\title{
Main-sequence and sub-giant stars in the globular cluster NGC 6397: The complex evolution of the lithium abundance
}

\author{
J. I. González Hernández ${ }^{1,2} \dagger$, P. Bonifacio ${ }^{1,2,3}$, E. Caffau ${ }^{1}$, M. Steffen ${ }^{4}$, \\ H.-G. Ludwig ${ }^{1,2}$, N. Behara ${ }^{1,2}$, L. Sbordone ${ }^{1,2}$, R. Cayrel $^{1}$, and \\ S. Zaggia ${ }^{5}$ \\ ${ }^{1}$ GEPI, Observatoire de Paris, CNRS, Université Paris Diderot; \\ Place Jules Janssen 92190 Meudon, France \\ email: Jonay.Gonzalez-Hernandez@obspm.fr \\ ${ }^{2}$ Cosmological Impact of the First STars (CIFIST) Marie Curie Excellence Team \\ ${ }^{3}$ Istituto Nazionale di Astrofisica - Observatorio \\ Astronomico di Trieste, Italy \\ ${ }^{4}$ Astrophysikalisches Institut Potsdam, An der Sternwarte 16, \\ D-14482 Potsdam, Germany \\ ${ }^{5}$ INAF - Osservatorio Astronomico di Padova, \\ Vicolo dell'Osservatorio 5, Padua 35122, Italy
}

\begin{abstract}
Thanks to the high multiplex and efficiency of Giraffe at the VLT we have been able for the first time to observe the Li I doublet in the Main Sequence stars of a globular cluster. At the same time we observed $\mathrm{Li}$ in a sample of Sub-Giant stars of the same B-V colour.

Our final sample is composed of 84 SG stars and 79 MS stars. In spite of the fact that SG and MS span the same temperature range we find that the equivalent widths of the Li I doublet in SG stars are systematically larger than those in MS stars, suggesting a higher Li content among SG stars. This is confirmed by our quantitative analysis carried out making use of $1 \mathrm{D}$ hydrostatic plane-parallel models and 3D hydrodynamical simulations of the stellar atmospheres.

We derived the effective temperatures of stars in our the sample from $\mathrm{H} \alpha$ fitting. Theoretical profiles were computed using 3D hydrodynamical simulations and 1D ATLAS models. Therefore, we are able to determined $1 \mathrm{D}$ and 3D-based effective temperatures. We then infer Li abundances taking into account non-local thermodynamical equilibrium effects when using both $1 \mathrm{D}$ and $3 \mathrm{D}$ models.

We find that SG stars have a mean Li abundance higher by 0.1 dex than MS stars. This result is obtained using both $1 \mathrm{D}$ and $3 \mathrm{D}$ models. We also detect a positive slope of $\mathrm{Li}$ abundance with effective temperature, the higher the temperature the higher the Li abundance, both for SG and MS stars, although the slope is slightly steeper for MS stars. These results provide an unambiguous evidence that the $\mathrm{Li}$ abundance changes with evolutionary status.

The physical mechanisms responsible for this behaviour are not yet clear, and none of the existing models seems to describe accurately these observations. Based on these conclusions, we believe that the cosmological lithium problem still remains an open question.
\end{abstract}

Keywords. Stars: abundances, fundamental parameters, Population II - Galaxy: globular clusters: individual: NGC 6397

$\dagger$ Present address: Dpto. de Astrofísica y Ciencias de la Atmósfera, Facultad de Física, Universidad Complutense de Madrid, E-28040 Madrid, Spain. Email: jonay@astrax.fis.ucm.es 


\section{Introduction}

The determination of the baryonic density from the fluctuations of the cosmic microwave background (CMB) by the WMAP satellite (Spergel et al. 2007, Cyburt et al. 2008) implies a primordial $\mathrm{Li}$ abundance which is $\log (\mathrm{Li} / \mathrm{H})+12=2.72 \pm 0.06$, at least 0.3-0.5 dex higher than the Li abundance determined in metal-poor stars of the Galactic halo (Spite \& Spite 1982).

Many different models of Li depletion have been proposed to explain discrepancy: (a) Piau et al. (2006) proposed that the first generation of stars, Population III stars, could have processed some fraction of the halo gas, lowering the lithium abundance; (b) other authors suggest that the primordial Li abundance has been uniformly depleted in the atmospheres of metal-poor dwarfs by some physical mechanism (e.g. turbulent diffusion as in Richard et al. (2005), Korn et al. (2006); gravitational waves as in Charbonnel \& Talon (2005), etc.); and (c) finally, it has been also suggested that the standard Big Bang nucleosynthesis $(\mathrm{SBBN})$ calculations should be revised, possibly with the introduction of new physics as in e.g. Jedamzik (2004), Jedamzik (2006), Jittoh et al. (2008), Hisano et al. (2009).

Here we present the determination of $\mathrm{Li}$ abundances of subgiant (SG) and mainsequence (MS) stars of the cluster NGC 6397. This work provides the first observations of the Li doublet in MS stars of a globular cluster.

\section{Observations}

We performed spectroscopic observations of the globular cluster NGC 6397 with the multi-object spectrograph FLAMES-GIRAFFE at the VLT on 2007 April, May, June and July, covering the spectral range $\lambda \lambda 6400-6800 \AA$ at resolving power $\lambda / \delta \lambda \sim 17,000$.

We selected subgiant and dwarf stars in the colour range $B-V=0.60 \pm 0.03$, which ensures that both set of stars fall in a similar and narrow effective temperature range (see Fig. 3 online in González Hernández et al. 2009a).

The spectra were reduced with the ESO pipeline and later on treated within MIDAS. We correct the spectra for sky lines and, barycentric and radial velocity. We typically combine 17 spectra of dwarfs and 4 spectra of subgiants to achieve a similar $\mathrm{S} / \mathrm{N}$ ratio between 80 and 130 in both sets of stars. The mean radial velocity of the cluster stars is $v_{r}=18.5 \mathrm{~km} \mathrm{~s}^{-1}$.

\section{Stellar parameters}

We derived the effective temperature by fitting the observed $\mathrm{H} \alpha$ line profiles with synthetic profiles, using 3D hydrodynamical model atmospheres computed with the $\mathrm{CO}^{5} \mathrm{BOLD}$ code. The details of this code are provided in Freytag et al. (2002) and Wedemeyer et al. (2004). The ability of 3D models to reproduce Balmer line profiles has been shown in Behara et al. (2009). In that work, the $\mathrm{H} \alpha$ profiles of the Sun as well as the metal-poor stars HD 84937, HD 74000 and HD 140283 are studied. Ludwig et al. (2009) also quantified, from a purely theoretical point of view, the difference between the effective temperatures derived from $\mathrm{H} \alpha$ fitting using $1 \mathrm{D}$ and $3 \mathrm{D}$ models.

We also derived the effective temperatures of MS and SG stars using 1D ATLAS 9 model atmospheres (see Kurucz 2005) and the same fitting procedure. In Fig. 1 we display the histograms of the effective temperatures derived for MS and SG stars using both $1 \mathrm{D}$ and $3 \mathrm{D}$ models. In the $1 \mathrm{D}$ case we got similar effective temperatures for both sets of stars. However, using 3D hydrodynamical models we obtained hotter temperatures by 

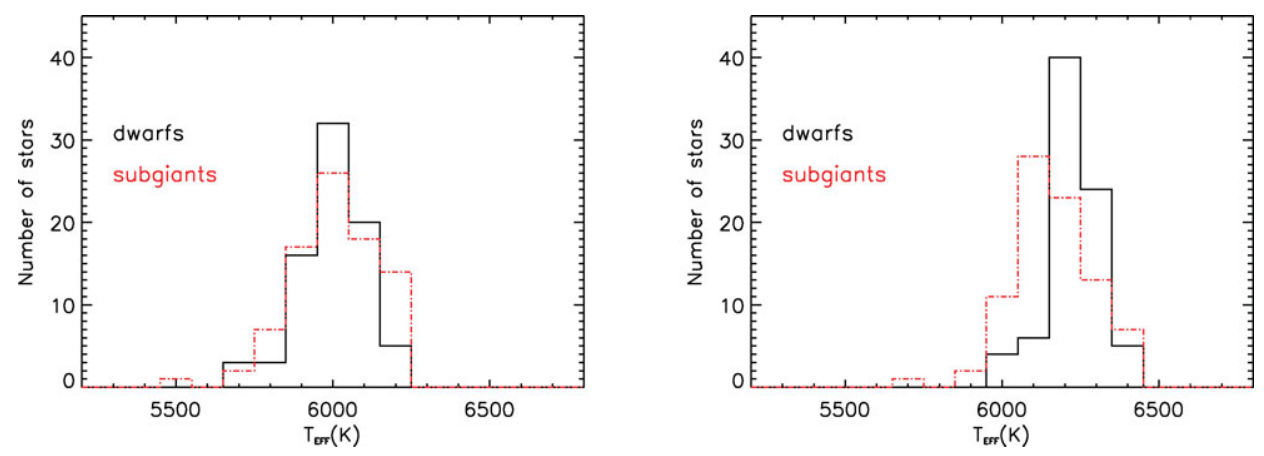

Figure 1. Histograms of 1D (left panel) and 3D (right panel) effective temperatures derived by fitting the observed $\mathrm{H} \alpha$ profiles with theoretical profiles computed using 1D (left panel) and 3D (right panel) model atmospheres, in bins of $100 \mathrm{~K}$, in MS (solid line) and SG (dashed-dotted line) stars in the globular cluster NGC 6397.

approximately $250 \mathrm{~K}$ in MS stars and $150 \mathrm{~K}$ for SG stars. In the 3D case the histogram of SG stars is slightly shifted with respect to the histogram of MS stars to cooler temperatures, as expected from the difference in surface gravity between MS and SG stars and the sensitivity of the $B-V$ colour to the surface gravity.

Fixed values for the surface gravity were adopted for both subgiant and dwarf stars in the sample, according to the values that best match the position of the stars on a 12 Gyr isochrone (Straniero et al. 1997). The adopted values were $\log \left(\mathrm{g} / \mathrm{cm} \mathrm{s}^{2}\right)=4.40$ and 3.85 for MS and SG stars, respectively.

\section{Li abundances}

We measure the equivalent width (EW) of the Li I $6708 \AA$ line in SG and MS stars by fitting synthetic spectra of known EW to the observed Li profiles. González Hernández et al. (2009a) showed the histograms of the EWs measured in SG and MS stars of this cluster (see their Fig. 1). In that figure it is clearly seen that the EWs of SG stars are larger than those of MS stars. They also estimate the weighted mean EW of the SG stars, being $\sim 1.1 \mathrm{pm}$ larger than the weighted mean EW of MS stars. Although the colour $B-V$ is sensitive to surface gravity, a priori, this result was not expected, and clearly suggests that subgiants in this cluster have actually higher Li abundances than dwarfs.

We derived $\mathrm{Li}$ abundances using 3D model atmospheres. The line formation of $\mathrm{Li}$ was treated in non-local thermodynamical equilibrium (NLTE) using the same code and model atom used in Cayrel et al. (2007). The analysis was also done using 1D model atmospheres providing essentially the same picture, even when $T_{\text {eff }}$ in $1 \mathrm{D}$ are lower (see also Fig. 6 online in González Hernández et al. 2009a). In the 1D case we used the Carlsson et al. (1994) NLTE corrections.

\section{Discussion and conclusions}

In Fig. 2 we display the histograms of the derived 1D-NLTE and 3D-NLTE Li abundances of dwarf and subgiant stars of the globular cluster NGC 6397. In both 1D and 3D cases, the SG stars have on average larger amounts of Li content in their atmospheres than MS stars. The difference in the mean Li abundance of dwarfs and subgiants is $\sim 0.14$ dex in the $1 \mathrm{D}$ case and $\sim 0.07$ dex in the $3 \mathrm{D}$ case. This difference between the $1 \mathrm{D}$ and the $3 \mathrm{D}$ cases is due to the effective temperatures derived using $1 \mathrm{D}$ and $3 \mathrm{D}$ models, 

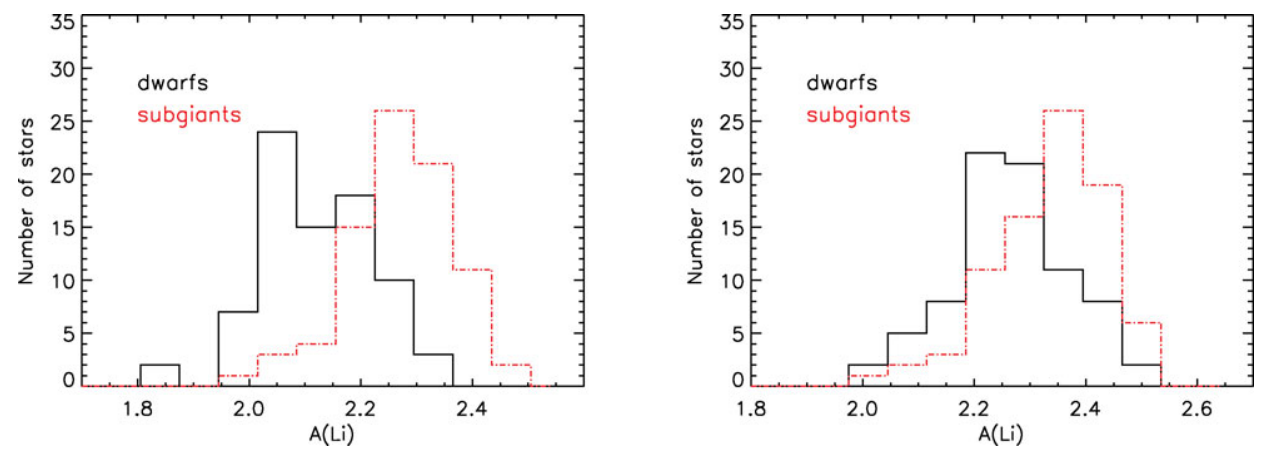

Figure 2. Histograms of 1D (left panel) and 3D (right panel) non-LTE Li abundances, in bins of $0.07 \mathrm{dex}$, in MS (solid line) and SG (dashed-dotted line) stars in the globular cluster NGC 6397.

and is not related to the NLTE corrections which were independently computed in the $1 \mathrm{D}$ and $3 \mathrm{D}$ cases. In fact, due to the cooler temperatures derived in $1 \mathrm{D}$ with respect to the $3 \mathrm{D}$ case, the mean $1 \mathrm{D} \mathrm{Li}$ abundance lower by $\sim 0.13$ dex for the MS stars $\sim 0.06 \mathrm{dex}$ for the SG stars than the mean 3D abundance.

Lind et al. (2009) also find different mean Li abundances, using 1D models, in MSs and SGs, but only by 0.03 dex although still significant at $1 \sigma$. However, their result is partially affected by the very narrow range of $T_{\text {eff }}$ for MSs deduced by Lind et al. (2009) $(\sim 80 \mathrm{~K})$ compared to the wide range $(\sim 450 \mathrm{~K})$ for the SGs (see Fig. 7 online in González Hernández et al. 2009a).

González Hernández et al. (2009a) showed, in their Fig. 2, the 3D-NLTE abundances of SG and MS stars versus 3D effective temperatures (see Fig. 1 in González Hernández et al. $(2009 \mathrm{~b})$ for a similar picture but with $T_{\text {eff }}$ and $\mathrm{Li}$ abundances computed using 1D models). The points in that figure display a decreasing trend of $\mathrm{Li}$ abundance with decreasing temperature. This lithium abundance pattern is different from what is found among field stars (see e.g. Meléndez \& Ramírez 2004, Bonifacio et al. 2007, González Hernández et al. 2008).

Our results imply that the Li surface abundance depends on the evolutionary status of the star. In Fig. 2 of González Hernández et al. (2009a), the Li isochrones are shown for different turbulent diffusion models (Richard et al. 2005). These models were shifted up by 0.14 dex in $\mathrm{Li}$ abundance to make the initial abundance of the models, $\log (\mathrm{Li} / \mathrm{H})=2.58$, coincide with the primordial Li abundance predicted from fluctuations of the microwave background measured by the WMAP satellite (Cyburt et al. 2008). The models assuming pure atomic diffusion, and, among those including low levels of turbulent mixing, in particular the model T6.0, preferred model in Korn et al. (2006) and Lind et al. (2009) are ruled out by our observations, since they predict the Li content in MS stars to be higher than in SG stars. The only model that predicts a Li pattern which is qualitatively similar to that observed, is the T6.25 model. This model shows a decreasing trend of $\mathrm{Li}$ abundances with decreasing temperatures and also predicts higher abundances for SG stars than for MS stars although at temperatures lower than $6000 \mathrm{~K}$, which is not consistent with the observed trend even in the 1D case (see Fig. 1 in González Hernández et al. 2009b).

Models including atomic diffusion and tachocline mixing (Piau 2008) do not seem to reproduce our observations either, since they provide a constant $\mathrm{Li}$ abundance up to $5500 \mathrm{~K}$. More sophisticated models are required, for instance, those models that besides including diffusion and rotation also take into account the effect of internal gravity waves 
(Talon \& Charbonnel 2004), seem to predict accurately the Li abundance pattern in solartype stars, at solar metallicity (Charbonnel \& Talon 2005), but models at low metallicity are still needed.

The cosmological lithium discrepancy still needs to be solved. Given that none of the existing models of Li evolution in stellar atmospheres matches our observations in the globular cluster NGC 6397, we hope our results will prompt further new theoretical investigations.

\section{References}

Behara, N. T., Ludwig, H.-G., Steffen, M., \& Bonifacio, P. 2009, American Institute of Physics Conference Series, 1094, 784

Bonifacio, P., Molaro, P., Sivarani, T., Cayrel, R., \& Spite, M. 2007, A\& $A$, 462, 851

Carlsson, M., Rutten, R. J., Bruls, J. H. M. J., \& Shchukina, N. G. 1994, A\& A, 288, 860

Cayrel, R., Steffen, M., Chand, H., Bonifacio, P., Spite, M., Spite, F., Petitjean, P., Ludwig, H. G., \& Caffau, E. 2007, A\&广A (Letters), 473, 37

Charbonnel, C. \& Talon, S. 2005, Science, 309, 2189

Cyburt, R. H., Fields, B. D., \& Olive, K. A. 2008, Journal of Cosmology and Astro-Particle Physics, 11, 12

Freytag, B., Steffen, M., \& Dorch, B. 2002, Astronomische Nachrichten, 323, 213

González Hernández, J. I. et al. 2008, A\&SA, 480, 233

González Hernández, J. I., Bonifacio, P., Caffau, E., Steffen, M., Ludwig, H.-G., Behara, N. T., Sbordone, L., Cayrel, R., \& Zaggia, S. 2009a, A\&A (Letters), 505, L13

González Hernández, J. I. et al. 2009b, Star Clusters: Basic Galactic Building Blocks Throughout Time And Space. Edited by R. de Grijs and J. Lepine. Proceedings of IAU Symposium \#266, held 10-14 August, 2009 in Brazil, Rio de Janeiro. Cambridge, UK: Cambridge University Press, in press

Hisano, J., Kawasaki, M., Kohri, K., \& Nakayama, K. 2009, Phys. Rev. D., 79, 063514

Jedamzik, K. 2004, Phys. Rev. D., 70, 083510

Jedamzik, K. 2006, Phys. Rev. D., 74, 103509

Jittoh, T., Kohri, K., Koike, M., Sato, J., Shimomura, T., \& Yamanaka, M. 2008, Phys. Rev. D., 78,055007

Kurucz, R. L. 2005, Memorie della Società Astronomica Italiana Supplement, 8, 14

Korn, A. J., Grundahl, F., Richard, O., Barklem, P. S., Mashonkina, L., Collet, R., Piskunov, N., \& Gustafsson, B. 2006, Nature, 442, 657

Lind, K., Primas, F., Charbonnel, C., Grundahl, F., \& Asplund, M. 2009, A $\& A$, 503, 545

Ludwig, H.-G., Behara, N. T., Steffen, M., \& Bonifacio, P. 2009, A\&A (Letters), 502, 1

Meléndez, J. \& Ramírez, I. 2004, ApJ (Letters), 615, 33

Piau, L., Beers, T. C., Balsara, D. S., Sivarani, T., Truran, J. W., \& Ferguson, J. W. 2006, ApJ, 653,300

Piau, L. 2008, ApJ, 689, 1279

Richard, O., Michaud, G., \& Richer, J. 2005, ApJ, 619, 538

Spergel, D. N., Bean, R. Doré, O., Nolta, M. R., Bennett, C. L. et al. 2007 ApJS, 170, 377

Spite, M. \& Spite, F. 1982, Nature, 297, 483

Straniero, O., Chieffi, A., \& Limongi, M. 1997, ApJ, 490, 425

Talon, S. \& Charbonnel, C. 2004, A\&A, 418, 1051

Wedemeyer, S., Freytag, B., Steffen, M., Ludwig, H.-G., \& Holweger, H. 2004, A\&SA, 414, 1121 


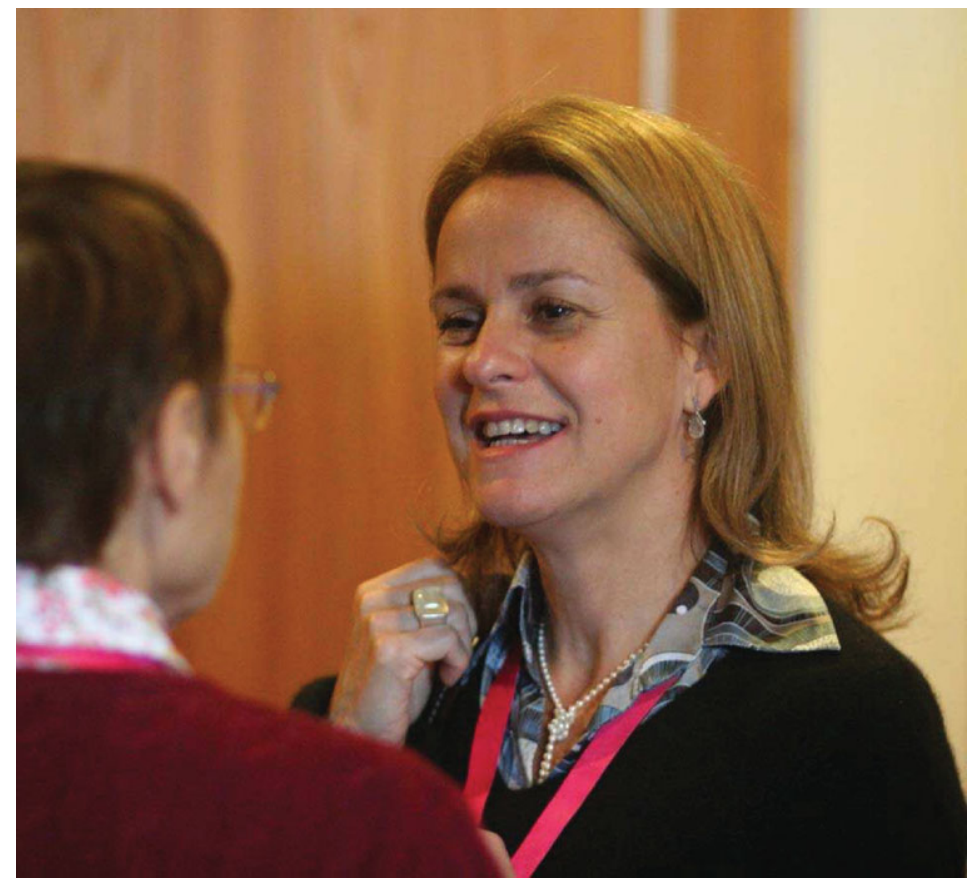

Monique Spite \& Katia Cunha

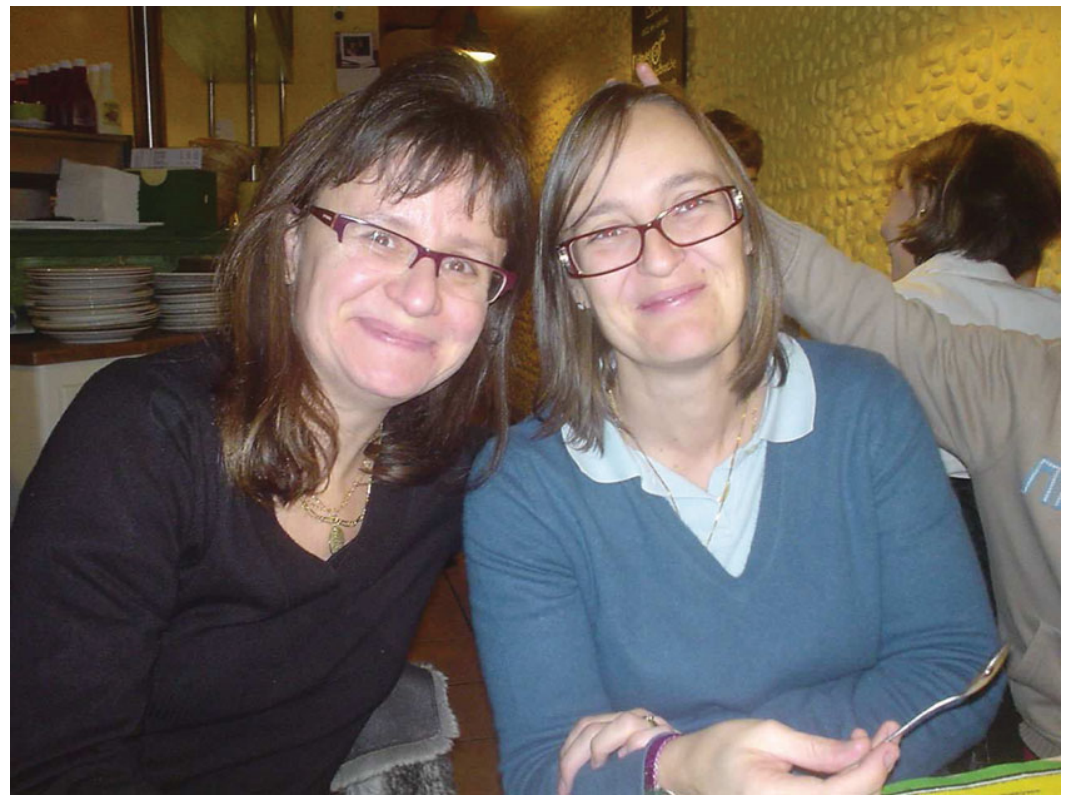

Corinne Charbonnel \& Francesca Primas 